\title{
Individual and ethnic aspects of preoperative planning for posttraumatic rhinoplasty
}

\author{
Pawel Szychta $\cdot$ Jan Rykała $\cdot$ Julia Kruk-Jeromin
}

Received: 10 April 2010 / Accepted: 10 August 2010 /Published online: 27 August 2010

(C) The Author(s) 2010. This article is published with open access at Springerlink.com

\begin{abstract}
The aim of this study was to compare the aesthetic results of post traumatic rhinoplasty among Europeans with populations of healthy Caucasians described in earlier reports, and to collate correct parameters of nasal shape in healthy representatives of both sexes and various races for improvement in aesthetic results of surgery. 3-D scanning of the face was performed in 54 patients after posttraumatic septorhinoplasty. Analysis of 3D model was based on two indices of the nasal proportions and four angles of the region from 18 anthropological points. Parameters of the nasal shape in addition to gender were compared to average values of healthy Caucasian population, described before. Normal characteristics of the nose among individuals of three races from previous studies were also compared to one another. In females, mean height and width of the nose as well as length of both nostrils was smaller. Neither were there any significant differences in width of the nostrils and length of the nasal pyramid nor nasal prominence. Nasal proportions were similar in both sexes. Some of the nasal angles differed in addition to gender. Posttraumatic rhinoplasty resulted in correct shape of the nose similar to the healthy Caucasian population. The aesthetic nose differ among healthy individuals of the three races analysed. While preoperative planning is important, knowledge of the normal values of parameters characterising shape in both genders is equally important as the individual differences in relation to the whole face.
\end{abstract}

Grants or other support None.

P. Szychta $(\bowtie) \cdot$ J. Rykała $\cdot$ J. Kruk-Jeromin

Plastic, Reconstructive and Aesthetic Surgery Department,

University Hospital No. 1, Medical University of Lodz, Poland,

ul. Kopciñskiego 22,

90-153 Łódź, Poland

e-mail: szychta@yahoo.pl
Keywords Posttraumatic · Distortion of nose Rhinoplasty · Sexual dimorphism · Caucasian race · Preoperative planning $\cdot$ Aesthetic nose

\section{Introduction}

Correction of posttraumatic distortion of the external nose is aimed as far as possible to restore the correct shape of the operated organ in order to obtain the "aesthetically perfect nose". The range of normal values of anthropometric parameters differs for males and females, and among Caucasian, Mongoloid and Negroid populations. Therefore, race and gender must be taken into account when carrying out nasal operations [1, 2].

While striving to obtain the correct proportions of the nose, the surgeon should plan the operation, noting that the nose is an integral part of the patient's face. Achieving harmony of the nose in accordance with parameters across the face, which is individual for each person, is one of the main aesthetic objectives of surgical correction [3].

The aim of this study is to compare the aesthetic results of posttraumatic rhinoplasty among Europeans with populations of healthy Caucasians as described in earlier reports. Moreover, the average values of the nasal shape will be collated among the representatives of both sexes and various races reported in the available literature to outline the clinically useful parameters for individual preoperative planning of rhinoplasty.

\section{Materials and methods}

The approval of the Review Board of the Medical University of Lodz RNN/57/10/KE was granted in order 
to do this research. In 54 patients enrolled into the study, there were 41 men and 13 women aged 18 to 45 years (mean age 27), all were treated surgically for posttraumatic distortion of the external nose and septum in the Department of Plastic, Reconstructive and Aesthetic Surgery, Medical University of Lodz, Poland, from June 2009 till March 2010. All patients included in the study had the following contour deformations of the nose: twisted nasal pyramid, dorsal hump, excessively protruding nose and significant tip rotation. The following surgical procedures were carried out in all cases: contour reconstruction of the posttraumatic nose, partial resection with repositioning of the nasal septum and fourfold osteotomy [4]. Nasal contour reconstruction consisted of: narrowing the nasal base, narrowing the nasal alae, decreasing the nasal projection, lowering the nasal tip rotation and removing the dorsal hump. The nose was stabilised with plaster of Paris and packing placed into both nasal cavities.

A three-dimensional model of each patient's face was created using a 3-D scanner after 6 months of surgery. A 3-D scanner based on structured light with an analytical module Antroposcan3D, developed by one of the authors (SP), was used in this study [5]. During the process of scanning, standardisation of the patient's position was not necessary, as, irrespective of the head's rotation, we obtained an identical three-dimensional model of the face, containing information concerning correct distances between any two anatomical points [6].

Analysis of the 3-D model was based on 18 anthropological points, including seven which were separately determined for the left and right side of the body, such as: subalar (sbal), alar (al) as well as points characterising nostrils: anterior (na), posterior (np), lateral (nl) and medial (nm; Fig. 1). Additionally, six isolated points of the nose were marked: pronasale (prn), subnasal (sn), nasion (n), glabella (gl), labiale superius (ls) and the so-called "columellar point" (cp). Using the selected anatomical points, eight linear measurements were done: length and width for both nostrils (respectively, naRnpR, naL-npL, nlR-nmR and nlL-nmL), nasal height (n-sn), length of nasal pyramid (n-prn), nasal width (al-al) and prominence of nose (sn-prn). Linear measurements were carried out with accuracy of about $0.1 \mathrm{~mm}$. The reliability of measurements using the anatomical points obtained by the 3D scanner has already been confirmed in previous studies $[7,8]$.

Computer analysis allowed us to obtain two indices of the nasal proportions and four angles of the region. Calculated linear indices included: index of prominence to width of the nose $($ sn-prn/al-al $\times 100)$ and nasal index $(\mathrm{al}-\mathrm{al} / \mathrm{n}$-sn $\times 100)$. The following angles were measured: interaxial angle (angle of the nasal tip, sbalR-cp-sbalL), nasofrontal (gl-n-prn), nasolabial (cp-sn-ls) and nasofacial (n-prn-sn) [9].

Parameters of the nasal shape obtained after surgery in men and women were compared with average values of a healthy Caucasian population described in previously reported studies. Proper characteristics of the nose among the representatives of three human races, described in the available literature, were also compared with one another $[6,10-14]$.

The significance of the differences between the values of the parameters in males and females was determined using statistical tests. Analysis of the normal distribution was carried out using Kolmogorov-Smirnof test and ShapiroWilk test. Subsequently, the appropriate tests were performed: Student's $t$ test for independent variables or MannWhitney $U$ test. Statistically significant difference was described as $p<0.05$. Data are presented as median \pm standard deviation (SD).

In order to avoid additional errors, the survey was conducted by the concept: one surgeon/one researcher/one photographer/one computer analyst [15].

\section{Results}

The shape of the nose differed between men and women (Table 1). In females compared with males, we found statistically significant lower values of the following linear parameters: height (respectively, $55.81 \pm 4.36 \mathrm{~mm}$ and $60.02 \pm$ $4.23 \mathrm{~mm}, p=0.0022)$ and width $(32.15 \pm 3.73 \mathrm{~mm}$ and $34.89 \pm$ $4.35 \mathrm{~mm}, p=0.0072)$. Length of both the right and left nostrils was smaller in women $(p=0.0457$ and $p=0.0207$, respectively), whereas, width of the nostrils in both sexes was similar $(p>0.05)$. Neither were there any significant differences in the length of the nasal pyramid (respectively, $45.66 \pm$ $5.58 \mathrm{~mm}$ and $49.02 \pm 6.60 \mathrm{~mm}, p=0.0560$ ) nor nasal prominence $(19.29 \pm 3.96 \mathrm{~mm}$ and $20.66 \pm 2.95 \mathrm{~mm}, p=0.2315)$.

Nasal proportions were similar in both sexes. Nasal index (width to height) was $57.72 \% \pm 5.93 \%$ in women and $58.25 \% \pm$ $6.92 \%, p=0.5257$ in men, while index of prominence to the width of the nose was $60.49 \% \pm 13.95 \%$ and $59.56 \% \pm 7.89 \%$, respectively $(p=0.4605)$.

As a result of statistical analysis, we found significant differences in both sexes for the following angles of the nose: interaxial (respectively, $69.02 \pm 11.89^{\circ}$ and $66.32 \pm$ $\left.13.21^{\circ}, p=0.0306\right)$ and nasofacial angle $\left(33.06 \pm 4.16^{\circ}\right.$ and $\left.31.64 \pm 3.01^{\circ}, p=0.0081\right)$. There were no significant differences for the nasofrontal $\left(132.95 \pm 5.99^{\circ}\right.$ and $131.05 \pm 5.10^{\circ}$, $p=0.4167)$ and nasolabial angle $\left(104.23 \pm 11.21^{\circ}\right.$ and $103.67 \pm 10.31^{\circ}, p=0.9530$ ).

\section{Discussion}

The ideal appearance of the face, as described by Leonardo da Vinci, is characterised by the presence of three equal parts, which are defined by four horizontal lines, respectively 
Fig. 1 Marked anatomical points used in the study together with indices of proportions (solid lines) and angles (dashed lines) shown on a sample 3-D model of the face in a patient after surgical treatment. View: a front, $\mathbf{b}$ profile, $\mathbf{c}$ from below
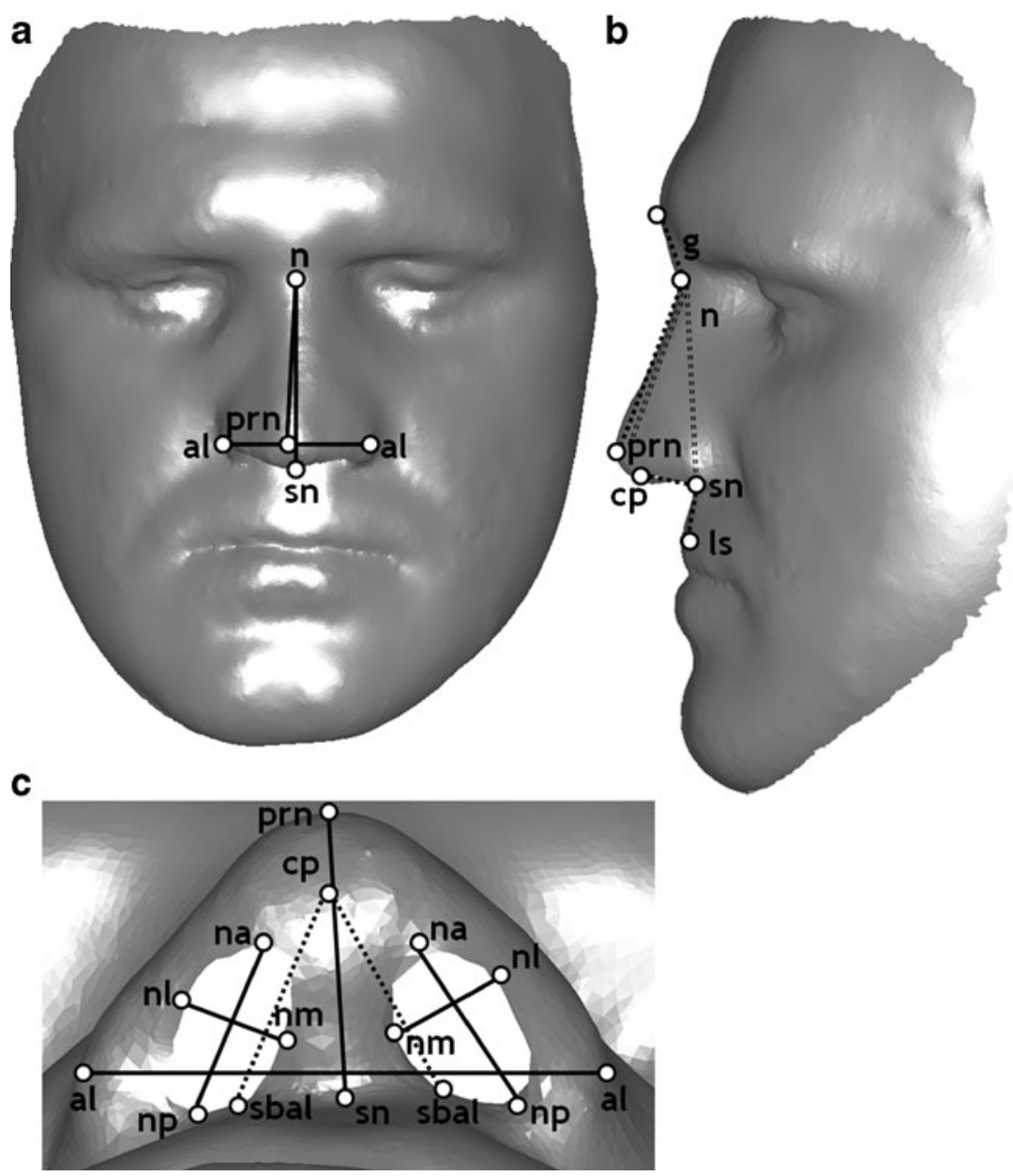

passing through the tip of the chin, nose, eyebrows (supraorbital notches) and medial part of the hairline [16]. According to new facial aesthetic canons, it should be divided into five equal parts marked by vertical lines passing through the tips of the ears, the outer corners of the eyes and the outer margins of the nose [17]. According to the above, the individual aesthetic appearance of the nose and the face depends integrally on each other. It should be noted, however, that in clinical practise, there is no universal concept of "perfect face" [18]. In contrast, the correct shape of the human nose, like the whole face, varies depending on race and gender [19].

Surgeons should be aware of differences in values of the anthropometric parameters of a normal nose in relation to gender, although there is no difference in the techniques of rhinoplasty in men and women [20]. Feminization of the male nose, associated with lack of understanding the differences in the shape of the nose in relation to gender, is not a rare complication of rhinoplasty. It should be noted that the nasion is in lower location in women compared to men [19]. The results obtained in previous studies were confirmed in our study where we observed narrower and shorter noses in female patients. Nostrils in women were rounder, with similar width and smaller length. For both sexes, we observed the same ratio between width and height of the nose and between its prominence and width. No difference in position of the nasal tip relative to the whole face was observed between both sexes. In conclusion, the nose was usually smaller in women.

Almost every human face in a healthy population has a significant mismatch between the two sides of the body. However, asymmetrical shape of the face may appear as interesting [21]. It is therefore important that the patient receives appropriate information concerning the possible postoperative deviations in shape from perfect symmetry after posttraumatic rhinoplasty [22]. In the current study, we obtained good results of corrective surgery of the external nose, which for most of the linear parameters, were similar to healthy Caucasian population. However, the nasal index was smaller than in the control group due to the narrowing of the nose performed in each case. Moreover, in the current study, slightly higher values of nasolabial angle were associated with surgical shortening of the nasal tip. Both decreased nasofrontal and nasofacial angles versus the control group were the result of osteotomy and the associated shift of nasion. 


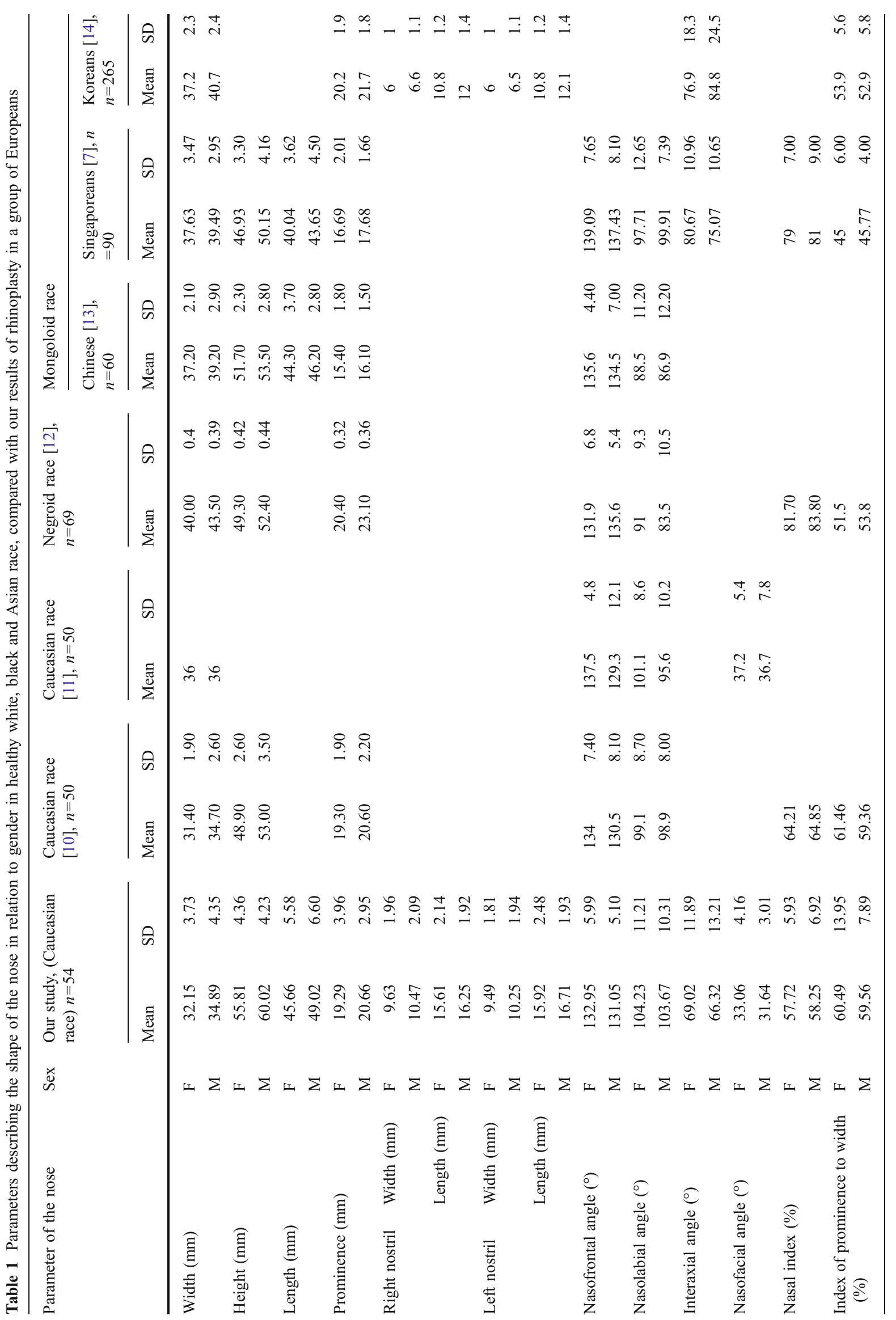


In the available literature, we found very few reports describing range of values defining parameters generally accepted in the "aesthetically ideal nose" [11]. Presently, many surgeons claim that certain facial proportions are judged as attractive in a specific cultural and ethnic population [23]. The shape of the nose is similar with the representatives of the Mongoloid race: Chinese, Singaporeans and Koreans (Table 1), with markedly more prominent nose in the last group [6, 13, 14]. Black people had the widest and most prominent nose compared with other ethnic groups. Caucasian nose is narrower than in Negroid and Mongoloid populations. It is also longer compared with other ethnic groups [24]. These findings are confirmed in our study. The average width of the nose was smallest in people of European origin. The length of the nose in Caucasian population was higher in relation to Mongoloid race. Europeans had also larger nostrils compared with those of Mongoloid race.

We claim that the "correct" range of values of parameters describing nasal shape should always include gender and race of the patient and the individual shape of the face. It should be remembered that the nose is an integral part of the face and must be adjusted in shape and size to the whole. Therefore, it is essential to obtain harmony among parameters of the nose during posttraumatic rhinoplasty. Even in healthy people with aesthetic appeal of the face, the number of parameters is usually different from the aesthetic standards [1]. A long or oval face looks attractive with a longer and narrower nose. Similarly, round or square face will be more harmonious with a shorter and broader nose [11]. During medical consultation, instead of being guided by "aesthetic ideal", surgeons should show understanding for the appropriate proportions of individual nose for each patient, treating the neoclassical canons only as a guideline. We think it is important to know the range of correct values for gender and ethnic group in order to obtain a perfect, individually tailored aesthetic result of the operation.

\section{Summary}

In our study of patients after posttraumatic rhinoplasty, we obtained an average shape of the nose similar to the healthy Caucasian population, which differed significantly compared with noses observed in healthy individuals of other races. While preoperative planning is important, knowledge of the normal values of parameters characterising shape in both genders is equally important as the individual differences in relation to the whole face.

Open Access This article is distributed under the terms of the Creative Commons Attribution Noncommercial License which permits any noncommercial use, distribution, and reproduction in any medium, provided the original author(s) and source are credited.

\section{References}

1. Rohrich RJ, Bolden K (2010) Ethnic rhinoplasty. Clin Plast Surg 37:353-370

2. Toriumi DM, Pero CD (2010) Asian rhinoplasty. Clin Plast Surg $37: 335-352$

3. Gryskiewicz JM, Hatef DA, Bullocks JM et al (2010) Problems in rhinoplasty. Clin Plast Surg 37:389-399

4. Dobratz EJ, Hilger PA (2010) Osteotomies. Clin Plast Surg 37:301-311

5. Antoszewski B, Szychta P (2008) System for measuring the surface of the human body and its modus operandi. Polish Patent Office P-386599

6. Aung SC, Foo CL, Lee ST (2000) Three dimensional laser scan assessment of the Oriental nose with a new classification of Oriental nasal types. Br J Plast Surg 53:109-116

7. Aung SC, Ngim RCK, Lee ST (1995) Evaluation of the laser scanner as a surface measuring tool and its accuracy compared with direct facial anthropometric measurements. Br J Plast Surg 48:551-558

8. Coward TJ, Watson RM, Scott BJ (1997) Laser scanning for the identification of repeatable landmarks of the ears and face. Br J Plast Surg 50:308-314

9. Sharp HR, Rowe-Jones JM (2003) Assessing outcome in aesthetic rhinoplasty. Clin Otolaryngol 28:430-435

10. Farkas LG (ed) (1994) Anthropometry of the head and face in medicine, 2nd edn. Raven, New York

11. Leong SCL, White PS (2006) A comparison of aesthetic proportions between the healthy Caucasian nose and the aesthetic ideal. J Plast Reconstr Aesthetic Surg 59:248-252

12. Ofodile FA, Bokhari F (1995) The African-American nose: part II. Ann Plast Surg 34:123-129

13. Farkas LG, Ngim RCK, Lee ST (1994) Craniofacial norms in 6-, 12-, and 18-year-old Chinese subjects. In: Farkas LG (ed) Anthropometry of the head and face, 2nd edn. Raven, New York, pp 201-218

14. Hwang TS, Kang HS (2003) Morphometry of nasal bases and nostrils in Koreans. Ann Anat 185:189-193

15. Muhlbauer W, Holm C (2005) Computer imaging and surgical reality in aesthetic rhinoplasty. Plast Reconstr Surg 115:20982104

16. Lines PA, Lines RR, Lines CA (1978) Profilometrics and facial aesthetics. Am J Orthod 73:648-657

17. Ozkul T, Ozkul MH (2004) Computer simulation tool for rhinoplasty planning. Comput Biol Med 34:697-718

18. Zhuang Z, Landsittel D, Benson S et al (2010) Facial anthropometric differences among gender, ethnicity, and age groups. Ann Occup Hyg 54(4):391-402

19. Springer IN, Zernial O, Nölke F et al (2008) Gender and nasal shape: measures for rhinoplasty. Plast Reconstr Surg 121:629637

20. Etöz BC, Etöz A, Ercan I (2008) Nasal shapes and related differences in nostril forms: a morphometric analysis in young adults. J Craniofac Surg 19:1402-1408

21. Meyer-Marcotty P, Alpers GW, Gerdes ABM et al (2010) Impact of facial asymmetry in visual perception: a 3-dimensional data analysis. Am J Orthod Dentofacial Orthop 137(168):e1-e8

22. Gruber RP, Roberts C, Schooler W et al (2009) Preventing postsurgical dissatisfaction syndrome after rhinoplasty with propranolol: a pilot study. Plast Reconstr Surg 123:1072-1078

23. Ducic Y, DeFatta R (2007) Closed rhinoplasty. Otolaryngol Head Neck Surg 18:233-242

24. Uzun A, Akbas H, Bilgic S et al (2006) The average values of the nasal anthropometric measurements in 108 young Turkish males. Auris Nasus Larynx 33:31-35 